Publications of the Astronomical Society of the Pacific

103: 1005-1011, September 1991

(C) 1991. The Astronomical Society of the Pacific. All rights reserved. Printed in U.S.A.

\title{
DIFFUSE-BAND OBSERVATIONS RELATED TO THE INTERSTELLAR EXTINCTION LAW
}

\author{
C. SNEDEN \\ Department of Astronomy and McDonald Observatory, University of Texas, Austin, Texas 78712 \\ A. WOSZCZYK \\ Institute of Astronomy, Nicolaus Copernicus University, Chopina 12/18, 87-100, Toruń, Poland \\ AND \\ J. KRELOWSKI \\ Leiden University, Laboratory Astrophysics, Postbus 9504, 2300 RA Leiden, The Netherlands \\ and \\ Institute of Astronomy, Nicolaus Copernicus University, Chopina 12/18, 87-100, Toruń, Poland \\ Received 1991 March 4, revised 1991 June 17
}

\begin{abstract}
This paper discusses the relation between the observed intensity ratios of the unidentified diffuse interstellar bands (DIBs) at $5780 \AA$ and $5797 \AA$ and the shape of the ultraviolet extinction curve determined along the same lines of sight. New high-resolution, low-noise spectroscopic observations are presented for nine lightly reddened stars chosen to exhibit very different ultraviolet extinction characteristics. It is shown that the strengths of these diffuse bands depend strongly on the extinction law, but that the intrinsic profiles do not; however, a physical interpretation of the observed phenomena is still very uncertain.
\end{abstract}

Key words: interstellar matter-extinction law-diffuse interstellar bands

\section{Introduction}

The diffuse interstellar bands (DIBs) have remained unidentified since their discovery in 1921-DIBs are thus the longest standing unsolved problem in all of spectroscopy. This fact is due partly to the typically very low signal-to-noise ratio of the spectra collected during the first 50 years of the DIB investigations. DIBs are typically rather shallow features and so their profiles (if not originating in optically thick media) are in many cases hidden in the noise. Moreover, diffuse bands, like any other interstellar absorption features, suffer Doppler splitting when observed in multiple-cloud lines of sight toward distant, heavily reddened stars. The advent of low-noise solid-state detectors has made possible the observations of DIBs in spectra of slightly reddened stars. These stars may be obscured by just single clouds. The obscuring media in such cases are much more likely to be homogeneous than the ill-defined averages formed along the lines of sight toward very distant but heavily reddened objects where all interstellar absorptions are usually very strong.

The first applications of high signal-to-noise spectra to the problem of DIBs have shown the diffuse spectra differing from cloud to cloud (Krelowski \& Walker 1987; Krelowski \& Westerlund 1988). It thus has become clear that diffuse bands are not of the same origin: the term diffuse includes simply all interstellar absorption features that are broader than, e.g., the $\mathrm{Na} \mathrm{D}$ doublet or the $\mathrm{H}$ and $\mathrm{K}$ lines and that are unidentified. The observed differences among the various lines of sight (Chlewicki et al. 1986, 1987) evidently show that interstellar clouds differ in their optical (and, thus, physical) properties. This fact may help in identifying DIBs - their behavior (related to that of some other interstellar features) may suggest a way to solve this fascinating problem.

The interstellar extinction law has been shown to be not identical toward different lines of sight. The complexities became evident when the first extraterrestrial UV spectra had been collected (Bless \& Savage 1972). The varying shape of the famous $2200 \AA$ bump and the strongly variable slope of the far-UV segment suggest varying physical properties of diffuse interstellar clouds. It was accidental (but lucky!) that the first two spectra (of $\sigma$ Scorpii and $\zeta$ Ophiuchi) showing very different ratios of the strong diffuse bands at $5780 \AA$ and at $5797 \AA$ (Krelowski \& Wes- 
terlund 1988) already had been shown to be associated with very different extinction laws.

Further investigations of the same objects demonstrated that the diffuse bands simply belong to the $a b$ sorption spectra of $\mathrm{H}$ I clouds and change together with other aspects of these spectra (Krelowski 1989). This conclusion was, however, based on an extremely small sample of observed stars. Moreover, the detailed investigations of the extinction curves brought forth another question that seems to be of basic importance: are the DIBs sensitive to all changes of the shape of extinction curve? Fortunately, enough satellite data exist nowadays to determine extinction curves of many early-type stars from their ultraviolet spectra. Two large catalogs of such extinction curves have been published already (Aiello et al. 1988; Fitzpatrick \& Massa 1990), presenting a pretty wide variety of possible extinction curves. A third catalog (Papaj, Wegner \& Krelowski 1991-hereafter PWK), based on the European Astronomical Satellite TD-1 spectra (e.g., Jamar et al. 1976), contains even more lines of sight with "peculiar" extinction curves (i.e., different from the "galactic average"-Seaton 1979; Savage \& Mathis 1979). This is because these spectra are of relatively bright and rather slightly reddened stars, thus more likely to be obscured by single clouds. In general, the homogeneous environments of single interstellar clouds are much more peculiarity-prone than long-distance averages over multiple clouds.

The present paper reports our initial attempts to find a detailed relationship between the observed peculiarities of the interstellar extinction law and the intensity ratio of the DIBs at $5780 \AA$ and $5797 \AA$ (hereafter called the 5780 and 5797 bands). From the data of PWK we have selected three stars with extinction curves resembling closely that of $\sigma$ Sco, three others with curves similar to $\zeta$ Oph, and three strongly peculiar Be stars, characterized by extinction curves completely lacking the $2200 \AA$ feature. In Table 1 the program stars are listed according to their membership in these "families". Table 1 also gives $E(B-V)$ reddening values, taken mostly from the catalog of Snow, York \& Welty 1977. The Snow et al. paper does not contain the stars HD 44458 and HD 45995. For these stars we estimated $E(B-V)$ from the published colors and spectral types (Hoffleit 1982), along with the recommended intrinsic colors of Schmidt-Kaler 1982. The $E(B-V)$ values quoted for all three Be stars should be treated with caution.

The plots of the ultraviolet extinction curves for the program stars are shown in Figure 1, in which we have divided the stars according to their membership in the $\sigma$, $\zeta$, and Be families. Note in particular the strong and "slim" $2200 \AA$ bump of the $\sigma$ lines of sight (Fig. la), the broad bump and far-UV rise of the $\zeta$ members (Fig. 1b), and the lack of the $2200 \AA$ feature and flat far-UV segment of the
TABLE 1

Program Star Data

\begin{tabular}{rcllll}
\hline \multicolumn{1}{c}{ HD } & Name & $\mathrm{E}(\mathrm{B}-\mathrm{V})$ & $\mathrm{S} / \mathrm{N}$ & $\tau_{5780}$ & $\tau_{5797}$ \\
\hline \multicolumn{5}{c}{$\sigma$ family } \\
40111 & 139 Tau & 0.145 & 350 & 0.071 & 0.034 \\
144217 & $\beta^{1}$ Sco & 0.210 & 575 & 0.077 & 0.025 \\
147165 & $\sigma$ Sco & 0.398 & 575 & 0.111 & 0.038 \\
& & & & & \\
& & $\zeta$ family & & & \\
23180 & $o$ Per & 0.315 & 550 & 0.044 & 0.077 \\
24398 & $\zeta$ Per & 0.339 & 625 & 0.038 & 0.075 \\
149757 & $\zeta$ Oph & 0.320 & 725 & 0.033 & 0.037 \\
& & & & & \\
& & $\mathrm{~B}_{e}$ family & & & \\
44458 & & $0.24:$ & 675 & 0.020 & 0.018 \\
45995 & & $0.16:$ & 700 & 0.011 & 0.007 \\
202904 & $v$ Cyg & 0.118 & 550 & 0.015 & 0.004 \\
\hline \hline
\end{tabular}

Be stars (Fig. 1c). These varied extinction curves clearly indicate that the intervening clouds are characterized by very different physical parameters. The very peculiar "bumpless" curves probably originate in circumstellar shells of Be stars. This may be suggested by the enormous value of the total-to-selective extinction ratio in the $\mathrm{Be}$ stars that follows from the usual curve-deriving procedure (Fig. 1c) - possibly caused by strong IR emission of grains situated in the close vicinity of these hot stars.

The question addressed here is whether a unique relation between the extinction law and the diffuse-band spectrum may exist. The diffuse interstellar bands, despite the slightly misleading adjective in their name, must be observed not only with high signal-to-noise ratio $(S / R)$ but also with high spectral resolution. Only with high resolution is it possible to investigate the profiles of the diffuse bands. The profiles are of basic importance, as some of the proposed DIB carriers (e.g., Cossart-Magos \& Leach 1990) relate them strongly to the physical conditions (temperature!) of the environment. Thus, we are interested not only in the presence or absence of DIBs, not only in estimates of their intensity ratios, but also in their widths and other details of profiles produced in extreme conditions. It already has been suggested (Westerlund \& Krelowski 1988a) that the single cloud profiles are always the same, irrespective of the observed intensity ratios and the extinction laws. This previous evidence did not sample, however, the extreme conditions of circumstellar shells, likely to be observed around Be stars. This is why we deliberately have selected a sample both of stars with normal and with strongly peculiar extinction 

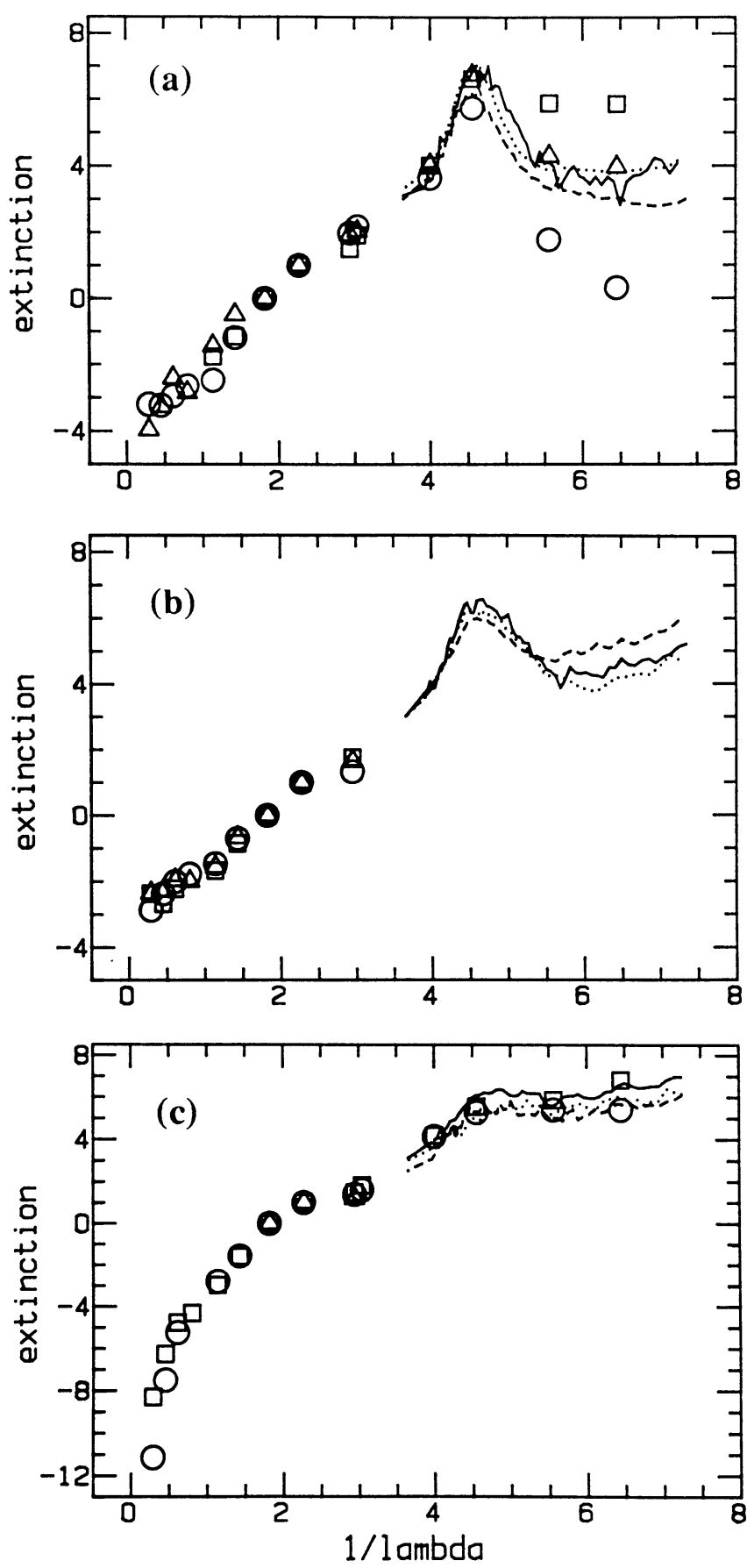

FIG. 1-The extinction curves of the target stars formed into three "families": (a) the $\sigma$ family (squares and solid line-HD 40111; triangles and dotted line-HD 144217; open circles and dashed lineHD 147165); (b) the $\zeta$ family (the symbols as in (a)) denote HD 23180 , HD 24398, and HD 149757, respectively); (c) the Be family (the symbols as in (a)) denote HD 44458, HD 45995; and HD 202904, respectively).

curves-if the DIB profiles still remain the same it is a very important constraint on the mechanism(s) of their origin.

\section{Observations and Reductions}

The spectra of the program stars were obtained with the McDonald Observatory 2.7-m telescope and coudé spectrometer. The spectrograph configuration utilized an ordinary reflectance grating as the dispersing element and a TI $800 \times 800$ CCD detector. This combination yielded spectra with linear dispersions of $0.066 \AA \mathrm{pixel}^{-1}$, as determined from a Th-Ar hollow cathode spectrum, and spectral coverages of approximately $53 \AA$. The spectrograph slit was set to project onto two pixels at the detector. The measured FWHM of the lines of the Th-Ar spectrum were about $0.17 \AA$.

To obtain high $S / N$ for the spectra, the stellar images were trailed along the slit, effectively increasing the number of photons captured to $\geq 10^{5}$ in all cases. This procedure eliminated photon shot noise as a major limiting factor in the $S / N$ of the reduced spectra. Probably more important were considerations of background subtraction and flat-field division. For background subtraction, first note that the program stars all are quite bright, which resulted in integration times less than 30 minutes even with the stellar image trailing. Also, the CCD chip was cooled to $-150^{\circ} \mathrm{C}$, so the background level was not large. Still, this weak background could be detected (beyond the bias offset signal of the CCD) and it exhibited a slow, repeatable variation from pixel to pixel, with the level being a direct function of integration time. Therefore, we gathered a large number of background frames with the integration times matched to the stellar exposure times. Likewise, multiple exposures of a tungsten lamp were obtained at the same instrumental settings as those for the stellar observations.

The initial reduction procedures of the CCD frames were carried out with the IRAF ${ }^{1}$ reduction package running on a microVAX computer at The University of Texas, Austin. With this software the following reduction tasks were performed: (a) subtraction of bias and trimming of the frames; (b) subtraction of the averaged (actually computed with a median) background frames; (c) division by the flat-field average frames; and (d) extraction of 1-dimensional spectra, with some filtering of the more obvious "radiation event" data spikes.

The final spectrum manipulations were done with special software (Fitzpatrick \& Sneden 1987) written for an IBMPC-AT computer. The first task involved division of the program-star spectra by those of hot, rapidly rotating stars (observed at approximately the same air masses as those of the program stars) that have essentially zero reddening and, hence, no detectable DIB features. This was done for three reasons. First, there are some ex-

${ }^{1}$ IRAF is distributed by National Optical Astronomy Observatories, which is operated by the Association of Universities for Research in Astronomy, Inc., under cooperative agreement with the National Science Foundation. 
tremely weak telluric water-vapor lines in this spectral region, but they are not negligible for our work. Second, the flat-field divisions via tungsten lamp frames exposed in the spectrograph did not remove the pixel-to-pixel sensitivity variations perfectly. This probably is due to the slightly different light paths of stars and lamp in the spectrograph. Third, the flat-fielding division still left a small residual curvature in the spectra that seemed to repeat (approximately) in the various spectra taken on a given night. The expense of a slight increase in the noise level of each pixel was considered a reasonable price to pay for the elimination of $\mathrm{H}_{2} \mathrm{O}$ features and the increased flatness of the spectra on short and long pixel scales. After these steps we estimated the $S / N$ ratios of all spectra by examination of the pixel-to-pixel variations in spectral regions away from the diffuse bands. These $S / N$ ratios are given in Table 1.

We next smoothed the data. A uniform smoothing was desired for all spectra, so we computed running means of every three pixels $(0.2 \AA)$. The DIB features are, of course, quite broad: the approximate FWHM of the 5780 band was $2.3 \AA$ and that of the 5797 band was $1.0 \AA$, as measured on our spectra (the profile widths seem also to be similar in all cases under consideration; see Section 3). Thus, the DIB profiles proved to be fairly insensitive to the particular smoothing algorithm adopted for this procedure.

Finally, a wavelength scale was implanted on the spectra. The basic (linear) dispersion was set by the Th-Ar spectrum. We then shifted the program-star spectra onto a consistent velocity frame by declaring the deepest point of the 5780 band in each spectrum to be a defined wavelength of $5780.80 \AA$. This wavelength is the rest central wavelength of the dominant component of the intrinsic profile of the 5780 band (Westerlund \& Krelowski 1988b). The final spectra of the two DIB features for all stars are shown in Figure 2, in which we have organized the panels ( $a, b, c)$ by membership in the three extinction families discussed in the Introduction. The obvious impression is that, as expected from earlier discussion, the intensity ratios of the features under consideration differ considerably from one family to another.

Since these spectra represent the first obtained with the McDonald Observatory 2.7-m coude spectrograph for this program, some comparisons were made with the results of previous investigators. To accomplish this we measured the equivalent widths of both bands in the program stars, using Simpson's Rule approximations for the calculations, and we searched the literature for published equivalent widths for these stars. We limited our search to papers with spectra from low-noise electronic detectors. Caution should be exercised in the interpretation of these comparisons, for the 5780 band and to a lesser extent the 5797 band lie in the midst of another DIB, usually called the $5778 \AA$ band (Herbig 1975). This

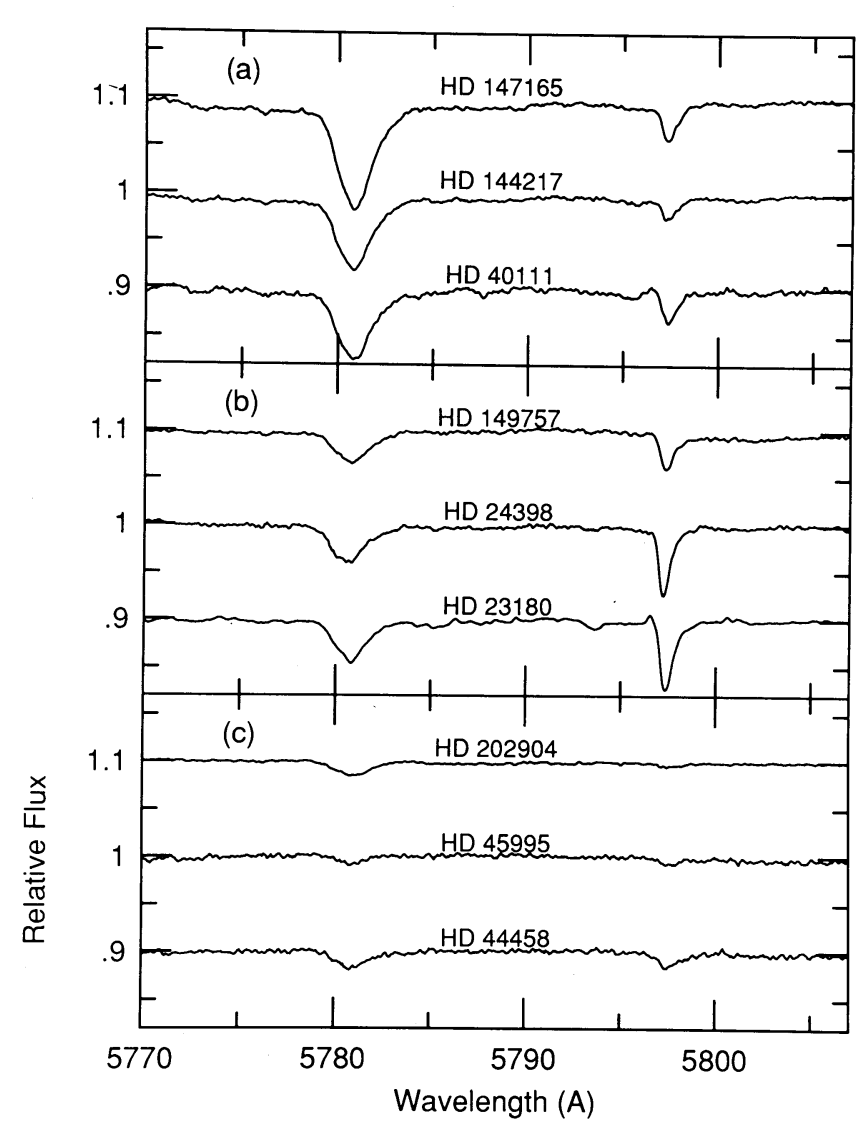

FIG. 2-The diffuse interstellar bands 5780 and 5797 observed in our targets, grouped for the panels by the same three "families" as in Figure 1. Note the strongly varying ratio of the bands. In each panel the relative intensity scale of the middle spectrum is correct, and the scales for the upper or lower spectra have been shifted vertically by additive constants of 0.1 .

band is extremely broad (nearly $20 \AA$ ) but quite shallow. We decided to compute the equivalent widths relative to a pseudocontinuum defined by the 5778 band. Usually the 5778 band is quite weak in the spectra of our lightly reddened stars, but it is not entirely negligible, especially in the stars HD 24398 and HD 147165. The presence of the 5778 broad absorption creates some uncertainty in the central depths of the 5780 and 5797 bands (see below), but, more importantly, it makes the definition of the wavelength limits of the bands more difficult.

Bearing these cautions in mind we have decided to defer an extensive equivalent-width discussion until a later paper in which data from a large sample will be presented and a very uniform approach to equivalentwidth measurements may be undertaken. For now we note two simple comparisons. First, Josafatsson \& Snow 1987 included HD 147165 in their CCD-based 0.4 Å resolution spectra of several DIBs. They gave equivalent widths of $240 \mathrm{~m} \AA$ for the 5780 band and $32 \mathrm{~m} \AA$ for the 5797 band. Our own estimates of $236 \mathrm{~m} \AA$ and $35 \mathrm{~m} \AA$ are in excellent agreement with their values. Second, Krelowski \& Walker 1987 reported equivalent widths 
for these bands for HD 24398 and HD 40111. For HD 24398, their 5780 and 5797 values are $96 \mathrm{m \AA}$ and $71 \mathrm{~m} \AA$, respectively, while the present measurements are $88 \mathrm{~m} \AA$ and $59 \mathrm{~m} \AA$. However, their equivalent widths were relative to a more distant continuum rather than relative to the 5778 band, and if we renormalize the continuum in our spectra in that manner we compute $100 \mathrm{~m} \AA$ and $65 \mathrm{~m} \AA$, closer to their values. For HD 40111 Krelowski and Walker list $153 \mathrm{~m} \AA$ and $55 \mathrm{~m} \AA$ equivalent widths and we derive $150 \mathrm{~m} \AA$ and $27 \mathrm{~m} \AA$ for the 5780 and 5797 bands. Similar fudging of the continuum does not change the equivalent width for the 5780 band significantly and raises that of the 5797 band from $27 \mathrm{~m} \AA$ to at most $40 \mathrm{~m} \AA$, so the basic difference in observed equivalent widths remains for this band. Further spectra should be obtained of HD 40111 to resolve this discrepancy.

\section{Band Profile Comparisons}

In order to compare the profiles of the DIB features in each target, we rescaled the features to match those observed in the spectrum of HD 144217. The following procedure was employed. First, for all stars the optical depths $\left(=\ln \left[I_{\text {continuum }} / I_{\text {band }}\right]\right)$ at each wavelength of both band profiles relative to nearby continuum points were calculated. Consistent with the equivalent-width measurements discussed above, we chose to measure the depths of the 5780 bands with respect to the underlying 5778 bands. This ignores the question of whether or not the 5778 band provides a true continuum for the 5780 band. We estimate that the error introduced into the optical depth at the 5780 band center by our use of the 5778 band as a continuum is less than $1 \%$ in all cases. In Table 1 we list the central optical depths for both bands in the program stars, where the central point is considered to be the deepest point of the band. From comparison of repeated observations of a single star we estimate the typical error in the optical depth to be \pm 0.002 .

Then, to intercompare the band optical depth profiles in different stars, each profile was scaled to match the maximum optical depth of HD 144217 (arbitrarily chosen as the reference spectrum). In Figure 3 we show these renormalized band profiles for the 5780 band, and in Figure 4 the results of this procedure are given for the 5797 band. Some very small wavelength shifts for the spectra to produce Figure 4 were made, but the extreme weakness of this band in our program stars precluded any meaningful investigation of the wavelength consistencies here. Note that the central depth of the 5780 band in HD 144217 is the second largest of all the program stars. Therefore, the scaling procedure effectively exaggerated the apparent noise of most other profiles after renormalization, as can be seen easily in Figure 3. However, HD 144217 does not have a particularly deep $5797 \AA$ feature, and so often the noise level of other profiles (see Fig. 4) was muted after renormalization.

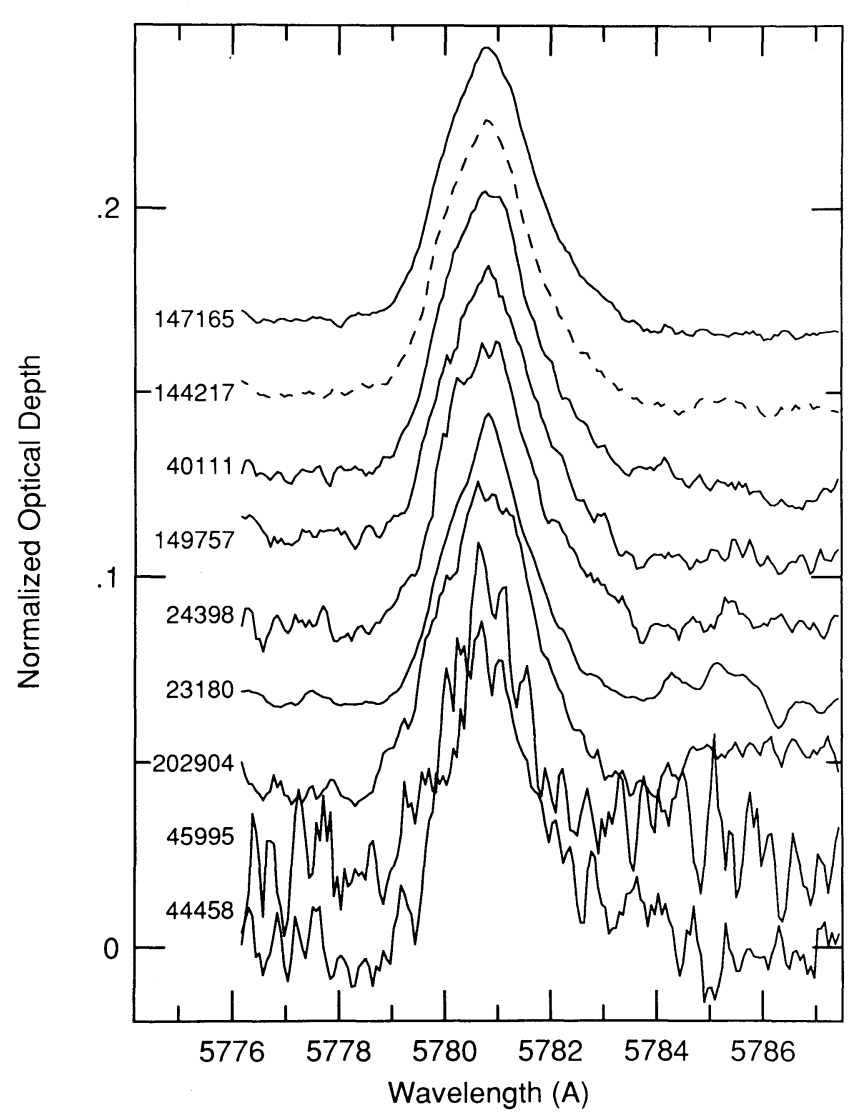

FIG. 3-The optical-depth profiles of the 5780 band in the program stars. The profiles all have been normalized to the central optical depth of the band in HD 144217 (dashed line). Note the excellent matches of all the profile widths, for the low S/N observation of HD 45995.

The 5780 and 5797 band profiles appear to be very similar in all lines of sight. Inspection of the profiles that have been plotted on top of one another reveals no variations in the shapes or half-widths in excess of the observational noise in almost all cases. The optical depth profiles exhibited in Figure 4 do suggest that small emission wings may exist at the blue edges of the 5797 bands in the stars HD 23180 and HD 40111. At present we doubt the reality of these features, for the continua in these spectra have more variations than are seen in most of the other program stars. We are gathering additional spectra of these stars to investigate this question further and also are obtaining more data for HD 45995 and HD 44458, whose DIB spectra are extremely weak.

\section{Discussion}

The observational data presented above provide additional evidence that different components of absorption spectra of diffuse interstellar clouds are related. The differences of diffuse-band ratios in spectra of clouds belonging to the $\sigma$ and $\zeta$ families have been reported already (Krelowski \& Walker 1987; Krelowski \& Westerlund 1988). The present paper, however, gives for the first 


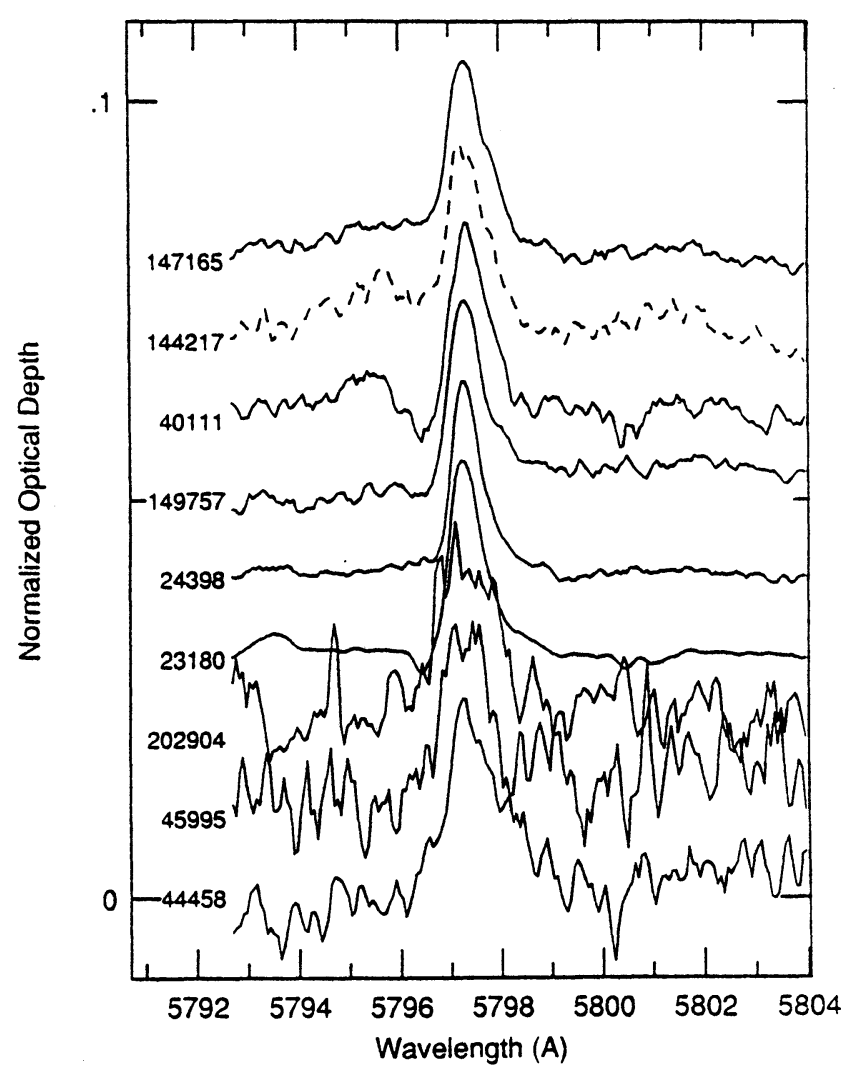

FIG. 4-The optical-depth profiles of the 5797 band compared to that observed in HD 144217 (broken line). The normalizations of the profiles are as in Figure 3. The profiles all are comparable (see comments in the text on the apparent emission features of HD 23180 and HD 40111).

time the spectroscopic observations on a uniform scale of lines of sight from all three broad categories of ultraviolet extinction curves. The uniformity of DIB strengths among stars of a given family is quite striking. For example, the star $\sigma$ Persei, chosen because of the similarity of its extinction curve to that of $\zeta$ Persei, fully confirmed our expectations for its DIB strengths. The ratios of DIBs in our third "family" resemble neither those of the $\zeta$ nor those of $\sigma$ cases.

Let us emphasize once again that the interstellar spectra observed in our cases are formed most probably in just single clouds. Remember that the bands observed here are typically very weak. This is difficult to avoid, for the stars characterized by such nontypical extinction curves are usually only slightly reddened. The profiles of sharp interstellar atomic lines observed in these spectra are typically very narrow and symmetric, not revealing any Doppler splitting. This has been demonstrated explicitly for HD 147165, HD 144217, and HD 149757 by Westerlund \& Krelowski 1988a. For HD 23180 and HD 24398 this point is well proven by Hobbs 1974. For HD 40111 the published interstellar Ca II $\mathrm{K}$ line has a slight asymmetry, suggesting a blend of two components split by a velocity of $<10 \mathrm{~km} \mathrm{~s}^{-1}$ (Hobbs 1984). This velocity difference is less than 1 resolution element in our spectra and will make a negligible impact on the widths of the DIB profiles for this star. Similarly convincing proofs do not exist for our third "Be family", but peculiar extinctions can hardly originate in many clouds along the investigated lines of sight. They must be formed in rather homogeneous media: such extinction laws are never observed in more heavily reddened stars.

We also may conclude that diffuse bands are being produced in extreme conditions of circumstellar shells around Be stars. Furthermore, the shells around our targets are optically rather thin. Thus, the DIB carriers are strongly resistant to the energetic far-UV photons. This confirms the conclusion of Westerlund \& Krelowski 1989 and makes it more general. This fact is of really basic importance as many molecules, which could be proposed as the DIB carriers, can hardly survive in tiny and heavily irradiated circumstellar clouds.

Another important conclusion concerns the surprising constancy of the profiles of DIBs. They seemingly are always the same (possible exceptions have been noted in the preceding section) irrespective of whether they originate in optically thick or thin clouds, and the profiles seem insensitive to the form of the ultraviolet extinction law. The varying shape of the far-UV extinction curve apparently plays a decisive role in the creation or destruction of many chemical species - the strong differences of molecular abundances toward the above-mentioned $\sigma$ Sco and $\zeta$ Oph stars are well-known (Danks, Federman \& Lambert 1983), but the penetration of the far-UV photons does not change the shapes of single-cloud DIB profiles. This fact makes the PAH (polycyclic aromatic hydrocarbons) hypothesis quite unlikely, since according to the calculations of Cossart-Magos \& Leach 1990 the widths of spectral features formed in this mechanism should be very sensitive to the temperature of clouds. It is almost certain that the circumstellar shells are not of the same temperature as normal interstellar clouds. Thus, the diffuse bands may be stronger or weaker in relation to the optical extinction, but the only process that seems to change efficiently their observed profiles is still simply Doppler splitting.

The small sample size of our first survey of DIBs in different UV extinction families has permitted us to make qualitative statements on the variations of the DIB strengths with extinction properties, but a quantitative analysis must await a significant augmentation of the number of targets surveyed. Such observations are now underway and will be reported in a future paper, along with a study of the correlations of these band strengths with the strengths of molecular absorption features.

The authors are very grateful to Professor J. Mayo Greenberg for a careful reading of the manuscript 
and very helpful comments. We thank Kalpana Gilroy, Harriet Dinerstein, and Kim Venn for gathering some of the spectra. The anonymous referee contributed very helpful suggestions that improved the analysis of the data. This project has been supported partially by the Polish Academy of Sciences under grant RPB-R 1.11 and by the U.S. National Science Foundation under grant AST 89-14917.

\section{REFERENCES}

Aiello, S., Barsella, B., Chlewicki, G., Greenberg, J., Patriarchi, P., \& Perinotto, M. 1988, A\&AS, 73, 195

Bless, R., \& Savage, B. 1972, ApJ, 171, 293

Chlewicki; G., de Groot, M., van der Zwet, G., Greenberg, J., Alvarez, P., \& Mampaso, A. 1987, A\&A, 173, 131

Chlewicki, G., van der Zwet, G., van Ijzendorn, L., \& Greenberg, J. 1986, ApJ, 305, 455

Cossart-Magos, C., \& Leach, S. 1990, A\&A, 233, 559

Danks, A., Federman, S., \& Lambert, D. 1983, A\&A, 130, 62

Fitzpatrick, E., \& Massa, D. 1990, A\&AS, 72, 163

Fitzpatrick, M. J., \& Sneden, C. 1987, BAAS, 19, 1129
Herbig, G. 1975, ApJ, 196, 129

Hobbs, L. 1974, ApJ, 191, 381 1984, ApJS, 56, 315

Hoffleit, D. 1983, The Bright Star Catalog (New Haven, Yale Univ. Obs.)

Jamar, C., Macau-Hercot, D., Monfils, A., Thompson, G. I., Houziaux, L., \& Wilson, R. 1976, Ultraviolet Bright-Star Spectrophotometric Catalogue, ESA SR-27 (Paris, ESA)

Josafatsson, K., \& Snow, T. 1987, ApJ, 319, 436

Krelowski, J. 1989, in IAU Symposium 135, Interstellar Dust, ed. L. Allamandola \& A. Tielens (Dordrecht, Kluwer), p. 67

Krelowski, J., \& Walker, G. 1987, ApJ, 312, 860

Krelowski, J., \& Westerlund, B. 1988, A\&A, 190, 339

Papaj, J., Wegner, W., \& Krelowski, J. 1991, MNRAS, submitted (PWK)

Savage, B., \& Mathis, J. 1979, ARA\&A, 17, 73

Schmidt-Kaler, T. 1982, in Numerical Data and Functional Relationships in Science and Technology, New Series, Group VI, Vol. 2b, ed. K. Schaifers \& H. Voigt (Berlin, Springer-Verlag), p. 15

Seaton, M. 1979, MNRAS, 187, 73p

Snow, T., York, D., \& Welty, D. 1977, AJ, 82, 113

Westerlund, B., \& Krelowski, J. 1988a, A\&A, 189, 221 1988b, A\&A, 203, 134 1989, A\&A, 218, 216 